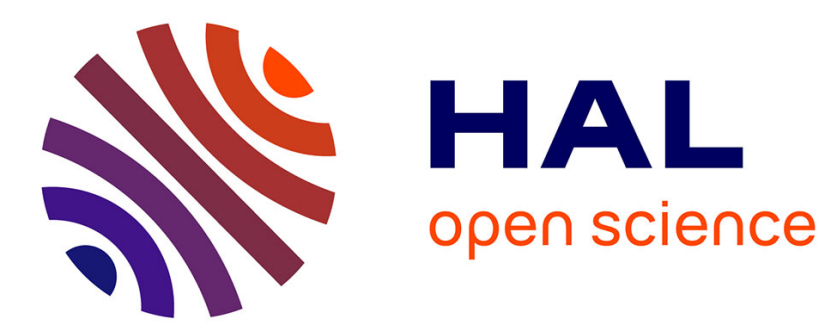

\title{
Le pilier et la corde: recherches sur la poésie maure
} Catherine Taine-Cheikh

\section{To cite this version:}

Catherine Taine-Cheikh. Le pilier et la corde: recherches sur la poésie maure. Bulletin of the School of Oriental and African Studies, 1985, XLVIII (part 3), pp.516-35. halshs-00456221

\section{HAL Id: halshs-00456221 https://shs.hal.science/halshs-00456221}

Submitted on 12 Feb 2010

HAL is a multi-disciplinary open access archive for the deposit and dissemination of scientific research documents, whether they are published or not. The documents may come from teaching and research institutions in France or abroad, or from public or private research centers.
L'archive ouverte pluridisciplinaire HAL, est destinée au dépôt et à la diffusion de documents scientifiques de niveau recherche, publiés ou non, émanant des établissements d'enseignement et de recherche français ou étrangers, des laboratoires publics ou privés. 


\section{LE PILIER ET LA CORDE: \\ RECHERCHES SUR LA POESIE MAURE}

\section{Par Catherine Taine-Cheikh}

Voici une dizaine d'années encore, bien peu de travaux avaient été consacrés à la poésie en Mauritanie ou du moins au gine, la poésie en dialecte hassāniyya. Nous n'avons toujours pas l'équivalent pour le gne du fameux al-Wasit que le mauritanien Sid Ahmed Ould Alamin publia en Egypte au début du siècle et qui comporte une anthologie du šr $r$, la poésie mauritanienne en arabe classique. Cependant, ces dernières années, beaucoup de poésies dialectales ont été recueillies et transcrites, quelques-unes sont publiées, d'autres devraient l'être ou ont fait l'objet de mémoires à l'École Normale Supérieure de Nouakchott (cf. bibliographie).

Mon but n'est pas ici de publier des poèmes inédits, même si quelques-uns le sont effectivement, mais de synthétiser et approfondir nos connaissances sur la versification. ${ }^{2}$ En effet, un examen attentif de toutes les données disponibles m'a amenée à des conclusions nouvelles sur la métrique du gine et sur ses rapports avec la métrique de la poésie en arabe classique.

Je ferai auparavant une description rapide des différents mètres et formes poétiques utilisés dans le ġne, mais commencerai, en guise de préliminaires, par clarifier l'analyse en mutaharrik et sākin exposée par Albert Leriche dans un article qui reste une référence très citée.

\section{Mutaharrik, sākin et syllabe}

1. Dans 'Poésie et musique maure', Leriche écrit que les vers maures sont formés de deux, trois ou quatre hémistiches composés d'une succession de syllabes accentuées ou brèves 'mutaharrik' et de syllabes longues 'sākin' (Leriche, Bull. IFAN, xII, 1950, p. 713). Reprenant les conventions en usage chez les Maures, il donne ensuite des exemples en représentant par un point - le mutaharrik et par une barre verticale | le sākin.

Les termes de mutaharrik et de sākin sont empruntés aux grammairiens arabes et ne se comprennent que par référence aux haurüf, les lettres de l'alphabet, et aux harakāt, les.voyelles brèves. Lorsque le harf, la lettre de l'alphabet, est accompagné d'une haraka (litt. 'mouvement') il est dit mutaharrik 'en mouvement', s'il ne possède pas sa haraka (l'absence de haraka étant marquée par la présence d'un sukūn, litt. ' repos '), il est dit sākin ' en repos'.

Leriche commet donc une erreur (erreur dont découle toute une série de contradictions dans la suite de l'article) en assimilant la syllabe longue au sākin, alors que le sākin correspond pratiquement à une lettre, une consonne seule. Par exemple, la préposition man ' de 's'analyse en un mutaharrik(ma-) suivi d'un sākin $(n)$ et se représentera par un point suivi d'une barre: $\cdot$.

L'analyse des grammairiens arabes est claire, à condition de bien voir qu'il ne s'agit pas d'une analyse syllabique. Le seul point qui fasse problème est celu

${ }^{1}$ En 1978 j'ai travaillé quelques semaines avec Dustin Cowell, chercheur américain qui menait alors une étude comparative sur la poésie amoureuse en Tunisie et en Mauritanie. S'il a publié l résultat de ses recherches, je n'en ai malheureusement pas eu connaissance. Depuis j'ai étudié avec Abdel Wedoud Ould Cheikh certains poèmes de Seddūm Ould Nd̆artu, dont le très célèbr rasm qui fera l'objet d'une étude conjointe.
2 Faute de place, je n'ai pu insérer dans

Pour la même raison les traductions données sont rédu texte tous les poèmes cités en exemple. toujours compte du sens littéral. du alif, du y $\bar{a}^{\prime}$ et du $w \bar{a} w$. En arabe, les voyelles longues $\bar{a}, \bar{\imath}$ et $\bar{u}$ s'écrivent de la même façon que les diphthongues ay et aw, par une haraka suivie d'une lettre aussi les grammairiens arabes n'ont-ils pas dissocié le alif, le yä' et le $w \bar{a} w$ des autres hurüf bienqu'ils soient conscients des rapports étroits existants entre la fatha et le alif ( $a$ et $\bar{a})$, la kasra et le $y \vec{a}\left(i\right.$ et $\bar{\imath} / y$ ), la damma et le $w \bar{a} w(u$ et $\bar{u} / w) .^{3}$ L'analyse et la représentation de lexèmes apparemment aussi différents que šavne 'nous avons vu ', žeyne ' nous sommes venus ' et kālu 'ils ont mangé ' sera donc identique: un mutaharrik + un säkin + un mutaharrik $\cdot \mid \cdot$. (Ces exemples sont empruntés au hassānivya mais il en serait de même en classique).

En arabe classique les mètres de la poésie diffèrent entre eux à la fois par le nombre de syllabes ou le nombre de mutaharrik qu'ils comportent et par la répartition des sākin dans le vers, l'absence de sākin après le mutaharrik correspondant à une syllabe brève, la présence d'un sākin, à une syllabe longue (le rythme de versification est donc un rythme de quantité).

Dans la poésie en dialecte hassāniyya, le nombre de mutaharrik par vers est également fixe, comme le fait remarquer Leriche au début de son exposé. Si la structure syllabique du dialecte était identique à celle de l'arabe classique, il suffirait de remplacer "...succession de syllabes brèves: mutaharrik et de syllabes longues: sākin' par 'succession de syllabes brèves: mutaharrik et de syllabes longues: mutaharrik + sākin'.

La confusion de Leriche est cependant significative et peut se comprendre comme une conséquence indirecte de l'absence du concept de syllabe chez les grammairiens arabes. Cette absence, Leriche ne l'a pas perçue, aussi a-t-i traduit les termes arabes comme s'il avait affaire à la problématique syllabique à laquelle il était habitué.

2. La seconde difficulté que rencontre le lecteur dans l'article découle du fait que la structure syllabique du hassāniyya diffère sensiblement de celle du classique.

Dans" son 'Cours de phonétique arabe' (Études de linguistique arabe, p. 118-19), Jean Cantineau énumérait trois points fondamentaux sur lesquels les dialectes modernes se différenciaient du classique

- la syllabe, dans les dialectes, peut commencer soit par un groupe de consonnes, soit par une voyelle.

- elle peut se terminer par deux consonnes, en finale comme à l'intérieur du mot, ce qui est pratiquement exclu en arabe classique. ${ }^{4}$

- les syllabes longues, du fait de la chute d'un grand nombre de voyelles, sont beaucoup plus nombreuses dans les dialectes qu'en arabe classique.

Nous aurons une idée de la structure syllabique du hassāniyya grâce à l'inventaire des formes et des fréquences des types syllabiques effectué par David Cohen à partir d'un corpus de 7,000 syllabes (Le parler arabe hassānīyya de Mauritanie, p. 83). Pour faciliter l'interprétation des résultats, les seize types que l'auteur présentait par ordre de fréquence sont réordonnés différemment.

— syllable brève : $10.26 \%$

(syllabe ouverte à voyelle courte)

$\begin{array}{lrr}\mathrm{A} & \mathrm{cv} & 5.25 \% \\ \mathrm{~B} & \mathrm{v} & 4.74 \%\end{array}$

C ccv $0.27 \%$

3 On pourra consulter, pour plus de détails, l'article ' haraka' dans l'Encyclopédie de l'Islam, IIr, p. 176-7 et le chap. vii du livre d'Abdelkader Méhiri sur Les théories grammaticales d' Ibn Jinnì. Les syllabes du type cric (ex. mād dans māddatun) sont les seules syllabes doublement longues susceptibles d'apparaitre en dehors de la pause mais les métriciens les considerent comme inacceptables à l'intérieur d'un vers. cf. Georges Bohas, 'Watid. Définitions, discussions et perspectives',
Analyses-Théorie, 1980-81, p. 56-7 et note 4, p. 78-9. 
- syllabe longue : $68.98 \%$

(syll. fermée ou à voyelle longue)

— syllabe ultra-longue : $20.58 \%$

(syll. doublement fermée ou fermée à voyelle longue)

— syll. doublement fermée à voyelle longue

Ce tableau fait ressortir très nettement la predominance des sylabes longues (presque $70 \%$ ) en hassāniyya. Il montre aussi le pourcentage important de syllabes ultra-longues $(20 \%)$, y compris par rapport aux syllabes brèves qui ne représentent qu'une syllabe sur dix, contre $45 \%$ en classique (Cantineau, 'Esquisse d'une phonologie de l'arabe classique', Etudes de linguistique arabe, p. 198).

On pourra objecter que la structure syllabique du hassāniyya se trouve modifiée en poésie du fait de l'affaiblissement des frontières de mot. ${ }^{\bar{z}}$ A travers l'exemple de trois vers (il s'agit des premiers vers d'un poème du grand griot Seddūm Ould Nd̆artu, consacré à Hennūn Ould Bü Seyf) on pourra constate que cette modification existe mais qu'elle se fait plutôt dans le sens d'une que cetation du nombre des syllabes longues, au détriment de celui des syllabes ultra-longues. Chaque vers est transerit deux fois, la première transcription reflétant la prosodie du mot (découpage syllabique respectant les frontières de mot), la seconde, la prosodie du vers (découpage syllabique en pieds ou unités métriques - le signe marque la liaison entre la fin d'un mot et le début d'un autre).

$c$ est mis pour syllabe courte, $l g$ pour syllabe longue et $u l-\lg$ pour syllabe ultra-longue.

1 vars e-vag-rās $n z \bar{u} \bar{u}^{c}$ e-geyl

ul- $\lg \mathrm{c} \lg$ ul- $\lg$ ul- $\lg \mathrm{c}$ ul- $\lg$

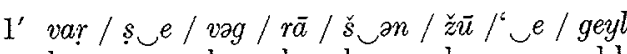

$$
\lg \mathrm{c} \quad \mathrm{lg} \quad \mathrm{lg} \quad \mathrm{lg} \quad \mathrm{lg} \mathrm{c} \quad \mathrm{ul}-\mathrm{lg}
$$

2 ax-yār al-hal-le w(e) et-yes-he

$\lg$ ul $\lg \lg \lg$ c $\quad$ (c) $\lg \lg \quad \mathrm{c}$

$2^{\prime} a x / y \bar{a} / r$ al / hal / le / wet / yes / he

$\mathrm{lg} \lg \lg \quad \mathrm{lg} c \mathrm{lg} \quad \mathrm{lg} \quad \mathrm{c}$

$3 m \bar{a}-h i$ varș e-vag-rāas bxīl

lg c ul-lg c lg ul-lg ul-lg 5 Il arrive même qu'un mot soit coupé en deux par la césure, qu'il commence à la fin d'un

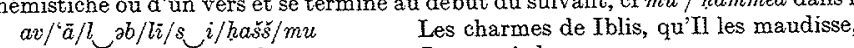

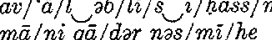

$m \bar{a}$ zow/le bü/he mant mu

ham/med ' $\bar{a} / \mathrm{li}$ mant $\mathrm{z} / \mathrm{h}$

Je ne puis les nommer,

hammed Ali de toute part. $3^{\prime} m \bar{a} / h i / v a r / s \smile e / v a g / r \bar{a} / \breve{s} \triangleleft a b / x \bar{a} l$

$$
\lg \quad \mathrm{c} \quad \mathrm{lg} \quad \mathrm{c} \text { lg } \mathrm{lg} \quad \mathrm{lg} \quad \mathrm{ul}-\mathrm{lg}
$$

La cavale du fier combattant de Egeyl,

Le premier de tous par ses vertus et sa témérité,

N'est pas la cavale d'un adolescent timoré (...)

On notera que la prosodie du vers tend à faire disparaître, sauf au début du vers, les svllabes du type $\mathrm{B}$, sans attaque consonantique (cf. evagräs et egeyl) et diminue considérablement le nombre des syllabes du type $\mathrm{C}$, à double attaque consonantique (cf. $n z \bar{z} \bar{u}^{\star}$ et $b x \bar{u} l$ ).

Si les syllabes, dans la poésie hassāniyya, commencent généralement par une consonne et une seule (alors que dans un usage non poétique de la langue, l'ensemble des syllabes de type $B$ représente $13.79 \%$ des syllabes et celui des syllabes de type $\mathrm{C}, 9.92 \%$ ), on pourra en déduire que la première différence entre la structure syllabique du dialecte et celle de l'arabe classique a disparu.

Cependant, puisque les deux autres différences sont confirmées (existence de syllabes ultra-longues et augmentation du nombre des syllabes longues en hassāniyya, comme dans l'ensemble des dialectes), on s'attendrait à ce que la conclusion à laquelle aboutissait Cantineau reste valable: 'La disparition d'un grand nombre de syllabes brèves (due à la chute de beaucoup de voyelles brèves en syllabe ouverte) a gravement altéré le rythme de quantité. Dans les parler maghrébins, où les syllabes brèves ont complètement disparu, le rythme de quantité a cessé d'exister puisque toutes les syllabes sont de durée comparable ' ('Cours de phonétique arabe', p. 121).

Il est fort possible que le rythme de quantité ait été altéré au point de disparaître dans la poésie au Maghreb, mais cela ne signifie pas que les syllabes sont devenues de durée comparable, puisqu'il existe dans tous les dialectes maghrébins des syllabes ultra-longues qui n'existaient pas en classique (cf. note 4). Si le rythme de versification demeure en hassanniyya un rythme de quantité, ce qui ressort parfaitement de l'étude de Leriche, c'est parce qu'une opposition devenait justement possible entre syllabe longue et syllabe ultralongue. Alors que le rythme de quantité se basait en classique sur l'opposition syllabe brève / syllabe longue (mutaharrik / mutaharrik + sākin), il se base en hassāniyya sur l'opposition syllabe brève ou longue / syllabe ultra-longue (mutaharrik ou mutaharrik + sākin / mutaharrik +2 sākin).

Ce déplacement de l'opposition, Leriche ne l'a pas perçu ou n'a pas su l'exposer clairement. Il ne dit même jamais explicitement que c'est le rencontre de deux sākin qui est pertinente et elle seule, ${ }^{6}$ et non la présence, comme en classique, d'un seul sākin. Aussi trouve-t-on dans son article quelques erreurs, en particulier dans les représentations des poèmes donnés en exemple (ainsi, dans le poème en mètre hweywìs, deux säkin représentés par deux barres verticales || apparaissent fautivement dans certains bémistiches, à la page 716) et dans le tableau récapitulatif des mètres à la page 728 (il représente les syllabes qui ne sont pas ultra-longues soit par un point, soit par un point suivi d'une barre alors que la répartition des mutaharrik et des mutaharrik + säkin ne peut être fixe puisqu'elle est, comme nous venons de le voir, non pertinente).

6 Selon la formulation de Md Mahmüd wuld 'Abd al-Fattäh wuld Abyayr reprise par Norris Folk lit., p. 41 et $156-7$ ).

La rencontre de deux sākin, les poètes la dénomment ged' $a$ (litt. ' arrêt brutal ' - - se dit en général des montures). Ils disent de l'hémistiche comportant une UM longue : vi-he ged"a, hiyye megdü $a$, mgenz'a (litt. 'assise en hauteur, haut perchee ) ou mkers' $a$ (hitt. accroupie, assise sur es talons ') alors que l'hémistiche sans UM longue est qualifiée de matlüsa (litt. 'libérée, lâchée ) ou mesrüme. 
Pour éviter une confusion dans l'expression de la quantité, je propose d'appeler unité métrique (UM) brève, l'unité métrique composée d'une svllabe brève ou d'une syllabe longue et unité métrique (UM) longue, l'unité métrique composée d'une syllabe ultra-longue. L'UM brève sera représentée par le signe u (qui correspondra à la fois au point · et à -1), l'UM longue sera représentée par le signe -, équivalent à $\cdot \|$.

3. J'aborderai une dernière question concernant l'identification des unités métriques du gne, celle de l'accentuation des unités longues. Personne, à ma connaissance, n'a soulevé ce problème, mais on ne s'en étonnera pas car la tradition arabe a systématiquement ignoré l'accent, ne le prenant en considération dans aucune de ses disciplines. De plus, le rôle et la nature de cet accent ne sont pas faciles à mettre en évidence.

Souvent l'unité métrique longue coincide avec la dernière (ou l'unique)

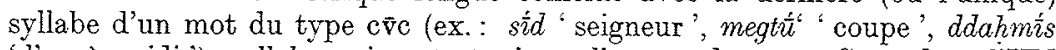
'l'après-midi '), syllabe qui porte toujours l'accent de mot. Cependant l'UMI longue peut correspondre aussi à la première syllabe d'un mot comportant une autre syllabe du type $c \bar{v} c$ ou $c \bar{v}(+c \bar{v})$ et l'UM longue ne porte pas alors l'accent de mot, ex.: nwākssút "Nouakchott', teyštáye, autre toponyme qui figure dans le poème en mètre sgayyar donné en III, C. S'il y a un accent sur l'UN longue, ce ne peut donc être l'accent de mot.

Considérons maintenant les deux hémistiches (ils constituent chacun le début d'un poème donné en entier dans la IVème partie de cette étude):
$\overline{d i}, l / l i b \bar{a} / d a t b-e \check{z} / l i$
Celle qui se joue de ma vie.

(début d'un mazzārag, composé dans le mètre la-bteyt an-nāqas qui ne comporte pas d'UM longue

met/qal $l$-oh $/ z \bar{z} / m \smile u \bar{d} \vec{\imath} \smile t / z ̌ \partial d d \quad$ 'Que la tristesse est éprouvante et celle-ci, on me le concèdera.

(début d'un poème du mètre laḅ̣̂eyr comportant une 6ème UM longue)

$d i$ ' celle-ci' figure dans les deux hémistiches mais la métrique nous oblige à transcrire différemment le démonstratif:

- dans le premier cas, seul $d i$ avec $i$ court est possible car autrement on aurait une UM longue, exclue dans ce mètre

- dans le second cas, le $\vec{\imath}$ de $\bar{\imath} \bar{\imath}$ est nécessairement long, faute de quoi l'hémistiche n'aurait pas d'UM longue au 6 ème pied.

On pourrait multiplier les exemples illustrant ce phénomène d' allongement' de la voyelle finale, centre d'une UM longue, et même donner une autre paire oppositive, avec le clitique $y a^{\text {' }} \hat{o}$ ' (qui se transcrit tantôt ya tantôt yā selon la nature, brève ou longue, de l'UM à laquelle il appartient).

Ce pourrait être l'effet d'une simple licence poétique s'il n'existait pas, dans le système phonologique du dialecte, des alternances de longueur comparables. En effet, en hassäniyya, les voyelles finales (à l'exception de la voyelle du féminin) sont des voyelles phonologiquement longues qui sont réalisées commes des courtes lorsqu'elles sont inaccentuées et ne retrouvent leur quantité de longue qu'en présence d'un suffixe qui fait reculer l'accent. Comparez: yélge vlän 'il rencontre qqn' et yelgâa-h 'il le rencontre', iméšš brắwe 'il envoie une

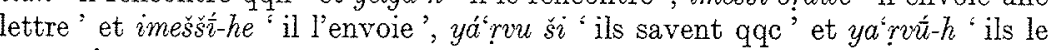
savent'.

La syllabe finale d'un mot n'est accentuée que s'il s'agit d'une syllabe ultralongue (cvcc ou $\mathrm{c} \overline{\mathrm{v}} \mathrm{c}$ ), dans les autres cas l'accent de mot remonte sur l'anté- pénultième. Un lexème monosyllabique qui n'est pas du type cvec ou cõc ne portera donc pas un accent de mot, à fortiori un clitique comme ya ' $\hat{o}$ '.

Il me semble que le $\bar{a}$ long de ya dans le vers à 1 ère UM longue (mètre bu 'amrān), y $\bar{a} \cup r / r a b / b \smile a l / b \partial^{\prime} /$ de man $h \bar{\imath} r . .$. " ô maitre, donne-moi l'éloignement de Hir...', tout comme le $\bar{\imath}$ long de $\bar{d} \bar{\imath}$ précédemment, est dû à la présence d'un accent que je qualifierai d'accent métrique, étant entendu que ce ne peut être un accent de mot.

La présence d'un accent métrique sur l'UM longue, distinct donc de l'accent de mot, a été pratiquement déduite par l'analyse, mais les résultats se trouvent parfaitement corroborés par la scansion (hikāye) des poèmes. En effet, la 'récitation' fait apparaître une nette distinction entre l'accent de mot qui est un accent d'énergie et l'accent métrique sur les UM longues qui est un accent d'intonation. ${ }^{7}$ L'UM longue correspond à une montée de la voix dont rendent compte métaphoriquement les termes maures appliqués à l'UM longue (cf. note 6): d'une part ged' $a$ signifie à la fois 'rencontre de deux sākin' et " note aigüe - en musique ', d'autre part genza' et kersa' ont en commun le sème 'en hauteur ' qui les distinguent d'un verbe comme $g^{\prime} a d$ ' s'asseoir'.

\section{Les différents mètres}

Les mètres (hass. bett, pl. btūte) se caractérisent donc par le nombre d'UM qu'ils comptent d'une part, par la présence ou non d'UM longue(s) accentuée(s), ainsi que leur place dans le vers, d'autre part. Ils comportent par ailleurs une rime en fin d'hémistiche (appelée rāâi en hasss.) que nous étudierons de manière plus approfondie dans le chapitre suivant.

Il s'agira essentiellement d'une synthèse rapide nécessaire à la suite de l'exposé. On trouvera les exemples qui n'ont pu figurer ici, faute de place, dans 'Poésie et musique maure' de Leriche et Shinqitit folk literature and song de H. T. Norris (désormais Folk lit.)

\section{A. Les mètres simples}

J'appelle mètre simple, le mètre dont les hémistiches sont absolument identiques. J'énumérerai les différents mètres en allant du plus court au plus long et je préciserai la nature des UM, sauf pour la dernière car elle correspond à la rime et sera étudiée dans le ch. III.

1. Le bett wähad (litt. 'le mètre de un') est un mètre, peu fréquent, où chaque hémistiche se compose d'une seule unité métrique. Leriche en donne un exemple à la page 718. (Md Ould Boyah, dans son mémoire intitulé La poésie de la résistance mauritanienne 1900-1933 lui donne également le nom de ġramz que je n'ai pu faire confirmer).

2. Le bett etneyn (litt. ' le mètre de deux ') - ou rsaym selon Md Ould Boyah (idem, p. 122) - comporte, comme son nom l'indique, deux UM par hémistiche, la 1 ère étant toujours brève, semble-t-il (cf. Leriche, p. 719 et Norris, p. 160-61).

3. Le bett etlāte (litt. 'le mètre de trois') comprend trois UM par hémistiche, les deux premières étant brèves. ${ }^{8}$

4. Deux mètres ont des hémistiches de quatre UM.

(a) hémistiche de 4 UM brèves, à l'exception de la dernière qui peut être brève

'Si l'on se réfère à l'analyse de Paul Garde (L'accent, en particulièrement le ch. III) on pourra peut-être mieux comprendre comment l'accent de mot et l'aceent métrique peuvent s'opposer en tant que procédés accentuels positifs (intensité/hauteur) tout en ayant en commun un procédé accentuel négatif (la perte des oppositions de longueur pour les voyelles en dehors de l'accent). Il aurait fallu disposer de deux signes differents pour représenter l accent métrique et l'accent do mot, ce qui ne semble pas, malheureusement, être dans l'usage.

a sweyr litt." celvi 
ou longue : uuuu/-. Ce mètre est appelé, en général, hwoeywīss. (D'après Leriche, cependant, hweywis compterait maintenant 5 UM et serait devenu synonyme de bteyt xamse.)

Exemple emprunté à El Kacem Ould Ahmedou (Soixante-cinq poèmes amoureux en hassanya, poème 14, page 16):

\begin{tabular}{|c|c|}
\hline 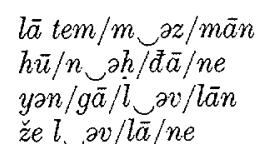 & $\begin{array}{l}\text { Si ce campement } \\
\text { Demeure près de nous } \\
\text { On dira que un tel } \\
\text { Est amoureux d'une telle }\end{array}$ \\
\hline
\end{tabular}

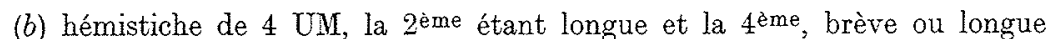
u-uu/-. Mohamed Ould Boyah appelle ce mètre mowmāyat ahweywīs (mowmāye est à rapprocher étymologiquement de twowme "gémir de douleur, de fureur, de ravissement...') et donne un poème en exemple (La poésie de la résistance, p. 21-2 et 119)

\section{$\partial s / s \bar{a} h / l, \partial x / s \partial r$}

$w \smile \partial t / v \bar{a} h \quad w \smile \partial d / m a r$

$w_{\smile} \partial l / x \bar{a} s / r \smile \partial k / t \partial r$

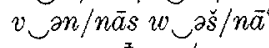

we xeyr de $z /$ zarr

$s \vec{a} / b a g m a \vec{a} \bar{a}^{6}$

$i /{ }^{\circ} \mathrm{amm} / \mathrm{l} \_\mathrm{s} / \mathrm{sarr}$

ye/kān ten $/ s \bar{a}^{\tau}$

$a n / s \bar{a} r / t \backsim a l / b a r r$

$w_{\smile a n / s \bar{a} r / t} \triangleleft \bar{d} / r \bar{a}$

L'ouest va mal

Il n'est plus que misère et ruine

Les agressions se multiplient

Et s'aggravent chez ces gens

Il serait préférable pour cette contrée

Avant sa totale destruction

Qu'elle use des sciences occultes

Pour chasser

Les Nazaréens de l'extérieur

Et ceux de l'intérieur

(II doit y avoir une petite erreur dans le 6 ème hémistiche car la 2ème UM y est brève, et non longue comme dans les autres hémistiches.)

5. Deux mètres au moins ont des hémistiches de 5 UM

(a) hémistiche de 5 UM brèves, à l'exception de la dernière, brève ou longue uuuuu/-. Ce mètre s'appelle bett xamse ("le vers de cinq '). 9

(b) hémistiche de 5 UII qui se distingue du précédent par la présence d'une 2 ème UM longue: u-uuu/-.

6. Le seul mètre courant de $6 \mathrm{UM}$ par hémistiche est celui qui ne comporte que des brèves à l'intérieur de l'hémistiche, d'où son nom de la-bteyt an-näqas

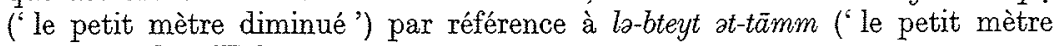
complet ') de 8 UM.10

7. Les hémistiches de $7 \mathrm{UM}$ sont ceux qui offrent le plus de variantes. (a) at-teydūm ("baobab'): hémistiche de 7 UM brèves, à l'exception de la dernière qui peut être brève ou longue uuuuuuu/-

(b) $b \vec{u}$ 'amrän: hémistiche de 7 UM avec une $1^{\text {ère }}$ (et éventuellement une dernière) UM longue -uuuuuu/-

9 Pour certains spécialistes, hatu žžrād est l'autre nom de bett xamse, pour d'autres comme Leriche hatu $z z r \bar{a} d$ designe un metre de 5 OM pouvant avoir une UM longue dans t'hemistiche. Miohamed Ould Boyah evoque le existence d un metre qui serait une variante du precédent, avec une 4ème UM longue : uuu-uu/.-. Un de mes informateurs, après m'avoir dit qu'à sa connaissance ce mètre n'existait pas en tant que tel, a improvisé un vers dans ce mètre: $m a t / m e n / n i$ ya $l m a / t a k$ Ne se comptent pas en ce moment.

Cela prouve qu'une $4^{\text {eme }}$ CM longue n'est pas impossible en soi mais aussi que tous les rythmes possibles n'ont pas la même valeur poétique aux yeux des poètes.

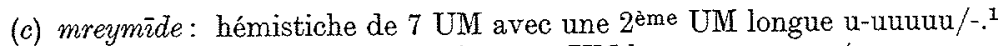
(d) tz̄gadrīn: hémistiche de 7 UM avec 5 UM longue uuuu-uu/-.

(e) la-bbeyr ('le petit puits'): hémistiche de 7 UM avec 6 ème UM longue uuuu-u/- La variante appelée tātrārt ou ntātrārt où les deux hémistiches sont rigoureusement identiques est beaucoup moins fréquente que la variante appelée bbeyr egilāl, la-bbeyr ou egilāl, que nous verrons plus loin. On trouvera un exemple du mètre ntätrārt chez Norris (Folk lit., p. 162-3).

8. la-bteyt at-tămm ("le petit mètre complet') est un mètre très fréquent. Il comporte 8 UM sans aucune UM longue, à l'exception de la dernière qui peut être brève ou longue uuuuuuuu/-. Selon certains, tous les mètres comptant moins de 8 UM peuvent être désignés sous le générique de la-bteyt 'le petit mètre', pourvu qu'ils ne comportent aucune UM longue à l'intérieur de l' hémistiche, comme la-bteyt at-tämm (cf. Norris, Folk lit., p. 41 et 158-9).

9. la-btüte la-kbār "les grands mètres': al-bett la-kbur (sg.) est un mètre de 8 UM dont une UM longue sans place fixe, ou un mètre de 9,10 UM, voire plus de $10 \mathrm{UM}$. On peut englober, sous le terme de $l a-b t u \overline{t e} l a-k b \bar{a} r$, d'autres mètre tels que al-wäkdi ou angādes (cf. Norris, Folk lit., p. 165). Je.parlerai peu de ces mètres, rares dans la poésie moderne et qui exigeraient une étude complémentaire.

\section{B. Les mètres complexes}

J'appelle mètres complexes les mètres dont chacun des deux hémistiches est composé dans un mètre différent, soit parce que le second hémistiche est plus court que le premier, soit parce qu'il lui manque une UM longue.

Pour faciliter l'exposé, je parlerai de $1^{\text {er }}$ et $2^{\text {nd }}$ hémistiche mais en fait il s'agit, en toute rigueur, des hémistiches de rime A par opposition aux hémistiches de rime $\mathrm{B}$, ce qui correspond, comme nous le verrons dans le chapitre suivant, à l'opposition $1^{\mathrm{e}}, 3^{\mathrm{e}}$ hém. $/ 2^{\mathrm{e}}, 4^{\mathrm{e}}$ hém. pour un gā $v$, mais à $1^{\mathrm{e}}, 2^{\mathrm{e}}, 3^{\mathrm{e}}, 5^{\mathrm{e}}$ hém. $/ 4^{\mathrm{e}}$ $6^{\mathrm{e}}$ hém. pour une talía.

Deux de ces mètres sont fréquents et j'en donnerai des exemples dans le ch. IV...

Le premier mètre complexe est bbeyr egilāl (ou egilāl, à rapprocher de mgeylel "à la queue coupée ') où le 2 ème hém. a une 6 ème UM brève à la place de la longue:

$$
1^{\mathrm{e}} \text { uuuuu-u } \quad 2^{\mathrm{e}} \text { uuuuuuu }
$$

Le second s'appelle sgayyar tz̄gadrīn ('le petit tägadrīn') souvent abrégé en sgayyar, parce que le premier hémistiche est identique à celui de tăgadrīn aveo une 5 ème UM longue uuuu-uu/- alors que le second hémistiche ne compte que 5 UM comme le bett xamse. Il arrive parfois que le second hémistiche soit de 6 UM au lieu de 5 , la 5 ème UM est alors longue comme dans le premier hémistiche (cf. ch. III).

Il existe un autre type de mètre alterné, très célèbre bien que peu fréquent, celui du Rasm. Le Rasm, créé par Seddūm Ould Ndartu, célèbre griot du 18 ème siècle, est un long poème mi-laudatif mi-épique dont l'une des carastéristiques est d'avoir des hémistiches alternés de 6 UM et 3 UM, la lère UM de l'hémistiche court étant une UM longue.

${ }^{11}$ Mohamed Ould Boyah affirme à la page 120 de son memoire que dans ce bett la dernière UM ne peut pas être longue comme dans les autres mètres. Son poème 25 lui offre cependant un démenti (rime $-\bar{\imath} l$ ):

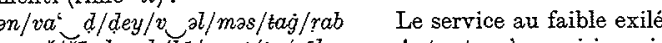

Autant qu'au fabisin puissant et exigeant.

Le début de ce poème figure également dans Le parler arabe hassāniza de Mauritanie, p. 238-9. 
Dans son mémoire, Mohamed Ould Boyah cite deux autres types de mètres alternés, l-mušsekkel (alternance $6 \mathrm{UM} / 5$ UM) et $l$-memlūx (alternance $5 \mathrm{UM} / 3$ UM). Signalons, sans nous prononcer sur l'existence de ces deux mètres, que d'après deux de nos informateurs, l-mušekkel n'est pas un nom de mètre mais désigne la rime croisée $A B A B$ (à rapprocher de škăl 'entrave croisée - d'une patte avant droite à une patte arrière gauche, ou l'inverse'), tandis que l-memlūx ou l-melx s'applique à l'hémistiche d'un poème, en particulier le dernier, lorsqu'il n'est pas du même mètre que les précédents.

Remarque: Le $t a b r \bar{a}^{c}$ est une forme à part, essentiellement pratiquée par les femmes. Une tabrî́a ne compte que deux vers, de même rime, en général d'inégale longueur. Souvent le $1^{\text {er }}$ vers est de 5 UM et le 2 ème de 8 UM, mais il existe peu de contraintes dans cette forme et il n'est pas rare que les poètes maures la considèrent avec un certain mépris (dédain affiché qui tient autant à la forme elle-même qu'au sujet - l'expression des sentiments d'une femme pour un homme-sujet qui outrepasse les règles sociales de la pudeur).

\section{La rime}

La présence d'une rime, non seulement en fin de vers mais également en fin d'hémistiche (pour ce qui est de la distinction vers/hémistiche, nous reprenons la tradition des lettrés maures), est une nécessité qui appelle peu de commentaires. De ce fait, rien ou presque n'a été dit la concernant alors que l'affirmation de son existence ne nous dit pas en quoi elle consiste ni comment elle fonctionne.

\section{A. Les différentes rimes}

Voici ce que donne, en pourcentage, un sondage portant sur 200 rimes:

1. $48 \%$ des rimes sont constituées par des syllabes ultra-longues telles que $-\bar{a} d,-\bar{a} n,-\bar{z} l$, etc. (à noter que les voyelles longues $\bar{u}$ et $\bar{\imath}$ alternent toujours librement avec les diphtongues ow et $e y$, respectivement, cf. egeyl et $b x \bar{l} l$ dans les trois vers donnés an ch. I, 2 alors qu'en classique une telle alternance constitue une violation des règles - un $\sin \bar{a} d$ ).

2. $19 \%$ des rimes comportent une voyelle longue suivie d'une syllabe brève ouverte $\bar{v} \mathrm{cv}$ telle que $-\bar{a} n e,-\bar{u} m e,-\bar{u} l u$ ou $-\bar{a} r u$.

3. $10 \%$ des rimes comportent deux consonnes suivies d'une voyelle brève ccv comme -tra, -lli, ou -bri. La voyelle qui précède les deux consonnes peut faire partie de la rime comme le a dans sāyab-he 'disposant d'elle', häzab-he 'la cloitrant' et žabhe 'front', mais ce n'est pas toujours le cas, cf. les rimes nasra 'majesté, hașra 'foule', kaṣra 'défaite' et mașra 'Égypte' (6ème et 4 ème poèmes du corpus d'El Kacem Ould Ahmedou).

4. $10 \%$ des rimes se composent d'une voyelle suivie d'une consonne ve, cf. -ar dans $x s a r$, dmar et ktar (voir au ch. II le poème en mètre mowmāyat ahweywīs). Parfois une partie de la syllabe précédente fait partie de la rime, ex. -ewwel dans lewwel 'premier', ntawwel 'je tarde', etc. A noter que la gémination de la consonne finale ne semble pas compter à la rime, ef. zarr et sarr rimant avec xsar et dmar dans le poème cité précédemment.

5. $7.5 \%$ des rimes se composent d'une seule syllabe brève ouverte $\mathrm{cv}$, ex. $\breve{s} i$ ' quelque chose ' rimant avec valši ' ma bien-aimée' ou ante 'toi ' avec hatte 'très'. Exceptionnellement une allitération vient renforcer la rime comme dans yankwe 'il est brûlé ' et yanšwe 'il est rôti'.

6. Enfin, dans $5.5 \%$ des cas, la rime est réduite à une seule consonne, $-d$ ou - $t$ le plus souvent: tantôt la consonne finale est toujours précédée d'une autre consonne, comme dans kant 'j'étais', 'att 'je suis devenu' et mant 'fllle', tantôt elle est précédée indifféremment d'une voyelle ou d'une consonne, ex. azrag 'tacheté ' rimant avec šarg 'Est'.12

Il existe une certaine hiérarchie entre les différents types de rimes. Un de nos informateurs nous accordait que les rimes du $1^{\mathrm{e}} \mathrm{t}$ type étaient supérieures à celles du 6 ème, mais il ne rejetait pas pour autant les dernières. Il semblerait que les rimes en $\overline{\mathrm{v}} \mathrm{c}$ ou $\mathrm{v} \mathrm{v}$, plus fréquentes que les autres, soient aussi jugées comme préférables. Les rimes les plus appréciées sont cependant celles où une homonymie cache une divergence de sens (le žinas), ex. ul ebmu "fils de Ebnou' et lebn-u 'son lait', 'ayn-he 'son œil ' et 'ayn-he (vìye) "elle (me) désire ' (ex. tirés des poèmes de Dustin Cowell).

$\mathrm{J}$ 'étais surprise de constater, dans le corpus qui me sert de référence, que le caractère emphatique ou non-emphatique des consonnes composant la rime était scrupuleusement respecté, y compris pour les couples $b / b, m / m, v / v$, $z / z, r / r, l / l$, ce qui confirmerait leur statut de phonèmes à part entière dans le dialecte hassāniyya. Ainsi, dans un poème de Mhammed Ould Ahmed Youra rapporté par Md Ould Ahmedou Bamba, vamm 'bouche' et kamm 'aile (d'une armée) 'riment avec deux noms de lieu se terminant par un $m$ emphatique (hsayyam et agžzm). Les poètes maures appellent zayy (litt. "cri, chant d'oiseau...') la rime qui ne respecte pas l'emphase ( $v \bar{\imath}-h$ zayy, litt. 'en lui, un cri ').

On remarquera que les seules rimes constituées d'une UM longue sont celles qui comportent une voyelle longue ou diphtongue suivies d'une consonne, ce qui r appelle l'arabe classique où 'les sur-lourdes qui apparaissent à la rime se limitent à: caac, cuuc, ciic, cayc, cawe'. (Georges Awad et Georges Bohas, 'Note à propos des sur-lourdes en arabe classique', Analyses-Théorie, 1981-1, p. 63.)

A la différence de la poésie en arabe classique, cependant, un vers peut très bien se terminer par une syllabe longue cvcc si la rime est constituée par une consonne seule (cf. ex. donnés ci-dessus en 5: kant, "att, mant) - sans parler du cas où la géminée n'est pas comptée comme dans zar(r) ou $\operatorname{sar}(r)$

J'ajouterai, pour terminer ces remarques sur les UM longues à la rime, que la dernière -UM de l'hémistiche, si elle est du type vic, ne porte pas un accent métrique d'intonation, mais un accent expiratoire, c'est à dire un accent de mot.

\section{B. Rimes et formes}

En dehors du tabrā de deux vers où la rime est unique $(A, A)$, il semble que tous les autres poèmes comportent au moins deux rimes dont les alternances dépendent de la forme poétique choisie.

\section{Le $g \bar{a} v$ (pl. $g \bar{\imath} v \bar{a} n$ )}

Le gāv compte quatre hémistiches en deux vers :

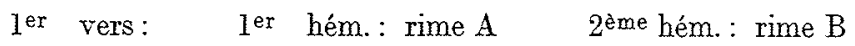

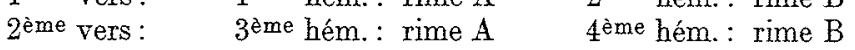

cf. ex. donné pour le mètre hweywūs en II, A, $4 a$.

Notons que d'après Leriche ('Poésie et musique maure', p. 715) le gāv msettet est un gāo de 3 vers (6 hém.) et le gãv ahmar, litt. 'gāo rouge', un gãa dont la rime est uniforme (variété peu prisée, semble-t-il).

12 Hamid Ben Alhousseini aborde le problème de la rime mais n'énumère pas tous les cas possibles - il ne cite aucun exemple des 1 , - (Aperçu sur la poésie maure de l'Azaouad Mali, ronéoté). 
La țal'a (pl. $\bar{a} t)$

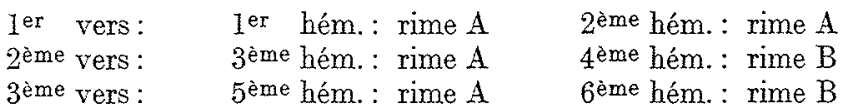

cf. ex. donné pour le mètre mowmāyat aȟweywīṣ en II, A, $4 b$.

Si la tal'a comprend plus de 3 vers, les 4 ème, 5 ème, 6 ème,.. vers sont sur le modèle du 3ème. J'ai trouvé beaucoup d'exemples de poèmes où une tal'a enchaine sur un gāv, un peu moins d'exemples illustrant la succession inverse. Dans les deux cas, la $2^{\text {ème }}$ rime est commune tandis que la lère change, on a done:

$\begin{array}{llll}t a l^{c} a & \text { A } & \text { A } & \text { Ier vers } \\ & \text { A } & \text { B } & \text { 2ème vers } \\ & \text { A } & \text { B } & \text { 3ème vers } \\ g \bar{a} v & \text { C } & \text { B } & \text { 4ème vers } \\ & \text { C } & \text { B } & \text { 5ème vers }\end{array}$

Le gā vet la tal'a sont les deux formes les plus fréquentes que l'on retrouve aussi bien dans la poésie amoureuse que dans d'autres genres, telle la joute poétique, la-gtă (cf. le mémoire de M. Abderrahim Ould Youra). Une définition stricte des genres poétiques, à la fois par la forme et le contenu, semble donc difficile; c'est une question que je ne résoudrai pas ici.

Le theydin (sg. theydinne, pl. $\bar{a} t$ )

La theydine est un long poème qui chante les louanges et les pronesses d'un grand personnage (en particulier guerrier). Composée dans une langue archaïsante, proche par son contenu de l'épopée, elle est l'œuvre des griots. Dans les trois theydināt présentées par Moulaye Ahmad Ould Hasni (La poésie épique hassane du Theydin), la rime est croisée comme dans le gā $v$ et les changements de rime se présentent toujours de la façon suivante: $\mathrm{A} B, \mathrm{~A} B, \mathrm{~A} \mathrm{~B},(\ldots), \mathrm{A} B$, A C , A C , (...) A C, D C, D C, D C, ... La plus grande partie du poème est cependant dans les rimes $\mathrm{A} B$.

\section{Le rasm}

Le contenu du rasm est le même que celui du theydìn et la langue comparable, voire encore plus archaïsante (le 'genre épique' compte donc au moins deux formes). Les spécialistes considèrent que le rasm compte quatre hémistiches, les trois premiers ayant la même rime et le quatrième, une rime différente identiques en cela aux quatre premiers hémistiches de la tal'a.

\begin{tabular}{|c|c|c|}
\hline $1^{\text {e }}$ vers & $\begin{array}{l}\text { Ier hém. }(6 \mathrm{UM}): \mathrm{A} \\
\text { 3ème hém. }(6 \mathrm{UM}): \mathrm{A}\end{array}$ & 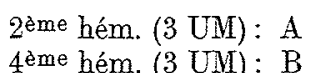 \\
\hline $2^{\mathrm{e}}$ vers & 1 ème hém. ( 6 UM) : A & 2 ème hém. (3 UM) : A \\
\hline & 3ème hém. (6 UM) : A & 4ème hém. (3 UM): \\
\hline
\end{tabular}

\section{Le mazzārag}

Le mazzōrag est une forme peu fréquente, composée de deux (ou plus?) vers de trois ou quatre hémistiches, rimant ainsi:

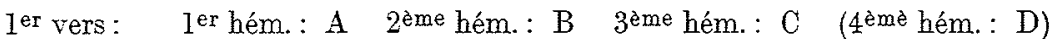

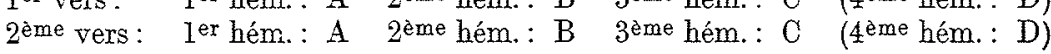

Norris donne un exemple de mazzārag à 3 hémistiches (Folk lit., p. 108-9). Voici un exemple avec 4 hémistiches:

\begin{tabular}{|c|c|c|}
\hline $1^{\text {er }}$ vers & 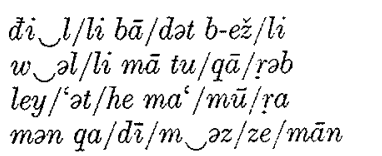 & $\begin{array}{l}\text { Celle qui se joue de ma vie } \\
\text { Et qui est inaccessible, } \\
\text { Cause de tourments infinis } \\
\text { Depuis les premiers temps, }\end{array}$ \\
\hline $2^{\text {eme }}$ vers & 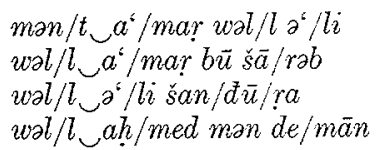 & $\begin{array}{l}\text { C'est la fille d'A'mar Ould E'l } \\
\text { Ould A'mar bū šārab } \\
\text { Ould E'li šanđūra } \\
\text { Ould Ahmed man Demān. }\end{array}$ \\
\hline
\end{tabular}

Le mazzärag me semble une forme et non un mètre, comme l'affirme Norris, bien que, dans son exemple comme dans celui-ci, chaque hémistiche soit identique à un hémistiche du mètre la-bteyt an-näqaș: il nous a été dit cependant que le choix de ce mètre n'était pas obligatoire.

\section{Rimes et mètres}

En arabe classique, l'étude des mètres et l'étude des rimes relèvent de deux sciences distinctes: "La théorie de la kāfiza est généralement considérée comme une science spéciale, différente du 'ilm al-'arud ou science de la métrique (...); elle enseigne comment doivent se terminer les vers en ce qui concerne les consonnes et les voyelles et quels sont les sons qui peuvent ou non apparaître à la rime' ('article ḳāfiya', Encyclopédie de l'Islam, rv, p. 429).

Ce n'est pas pour sacrifier à la tradition que j'avais jusqu'à présent séparé l'étude des rimes de celle des mètres, mais parce qu'effectivement rimes et mètres semblent le plus souvent indépendants l'un de l'autre. Si l'on considère, dans notre corpus de référence, l'ensemble des poèmes en mètre la-bteyt an-nāqas $(6 \mathrm{UM})$ et en mètre la-bteyt at-tâmm (8 UM), on se rend compte que toutes les rimes sont possibles: aussi bien dans les hémistiches de rime $A$, dans les hémistiches de rime $B$, que dans les diverses combinaisons possibles de la rime $A$ avec la rime $B(\overline{\mathrm{v}} \mathrm{c} / \overline{\mathrm{v}} \mathrm{c}, \overline{\mathrm{v}} \mathrm{c} /(\overline{\mathrm{v}}) \mathrm{cv},(\overline{\mathrm{v}}) \mathrm{cv} / \overline{\mathrm{v}} \mathrm{c},(\overline{\mathrm{v}}) \mathrm{cv} /(\overline{\mathrm{v}}) \mathrm{cv},(\mathrm{v}) \mathrm{c} /(\mathrm{v}) \mathrm{c}, \overline{\mathrm{v}} \mathrm{c} /(\mathrm{v}) \mathrm{c}, \ldots)$. Je ne pense pas m'avancer beaucoup en affirmant que le mètre la-bteyt au sens large (c'est à dire l'ensemble des mètres dont les hémistiches comptent au plus $8 \mathrm{UM}$, sans aucune UM longue dans l'hémistiche) n'exerce aucune contrainte sur la nature des rimes. Il semblerait que ce soit le cas également de la plupart des autres mètres, et en particulier de $b \bar{u}$ 'amrān (7 UM avec 1ère UM longue). Pour ce qui est du theydìn et du rasm, la rime ve y est prépondérante, mais toutes les rimes sont attestées, à l'exception de la rime -c (consonne seule).

Nulle part, semble-t-il, n'a été signalée l'existence de contraintes sur la rime $\mathrm{B}$ dans les mètres bbeyr egitäl et sjayyar (rappelons que la rime B est celle du $2^{\text {ème }}$ et $4^{\text {ème }}$ hém. dans le $g a \tilde{a} v$, mais du $4^{\text {ème }}, 6$ ème, ... hém. dans la tal' $a$ ).

Dans bụeyr egiläl, l'hémistiche de rime A comporte une 6 ème UM longue alors que celle de rime B n'en comporte pas: (A) uuuuu-u (B) uuuuuu.

met/qal lah $/ \bar{z} \bar{\imath} / m \smile u \bar{d} \bar{\imath} \smile t / z ̌ \partial d d \quad$ Que la tristesse est lourde, on me le con$l \partial h / \check{z} \tilde{\imath} / m 、 \partial l / g \bar{a} / t \partial^{c} m \bar{u} / l u$

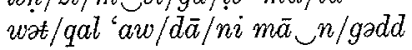
cèdera,

Et c'est d'autant plus pénible que je ne puis

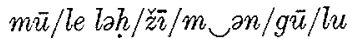

Quand s'estompe tout espoir de rencontre, Nommer l'objet de cette tristesse. 
$h \bar{a} / \bar{d} e \cup n / n e / s \bar{\imath} / m \cup \partial l / l \bar{t} t / q a$ man ga/ble $s \bar{a} / h a l=\bar{a} / h a l$ $k \bar{u} / n_{\smile} \partial^{\prime} /$ le had $/ d \_$, m $/$gowt/ro

Ce zéphir qui s'est levé

Du sud-ouest est bien étrange

Sauf pour celui que mènent à pas lents

Les chameaux se dirigeant vers l'ouest.

Alors que la rime $A$ est libre (l'un quelconque des 6 types recensés plus haut) la rime $B$ ne peut être que du type ṽcv (cf. -ülu dans le premier poème, cidessus) ou du type ṽcve (cf -ăhal dans le second poème). A la 6 ème UM longue de l'hémistiche de rime A correspond done toujours, dans l'hémistiche de rime B une UM - courte certes - mais comportant une voyelle longue, sur laquelle tombe un accent de mot.

Dans les poèmes de mètre sgayyjor, l'hémistiche de rime $B$, qui compte deux UM de moins que celui de rime A, a toujours une rime du type- - vc. Cette UM longue, comportant une voyelle longue (là encore toujours accentuée), correspond donc à la $5^{\text {ème }}$ UM longue de l'hémistiche de rime A. (Je n'ai pas trouvé de rime $A$ du type - $\vec{v} c$ mais hésite à faire de cette absence une règle, d'autant que cela est possible dans le mètre tägadrīn qui en est proche.)

\section{ag/bey/l ad/dah/mĩs ban/ti Un instant, au soir, tu es apparue $w a \cup b / l a g d \bar{a} / \dot{k} \cup a b / l \bar{s} s \quad$ Et Satan s'en est réjoui yah/rag bey/y ab/lizs w $\smile_{a n / t i} \quad$ Soyez damnés, toi, Satan, $w_{\smile a g / b e y / l} \_d / d a h / m \bar{\imath} s \quad$ Ce soir-là et cet instant.}

Dans le poème qui précède (poème 22 de El Kacem Ould Ahmedou) l'assonance interne en - $\bar{\imath} s-d d a h m \bar{\imath} s$, $\partial b l \bar{l} s$ - renforce la correspondance entre les 5 ème UM, mais ce n'est pas obligatoire comme on peut le voir dans le $2^{\text {nd }}$ exemple (poème 38 d'El Kacem Ould Ahmedou) où l'assonance n'existe qu'entre les

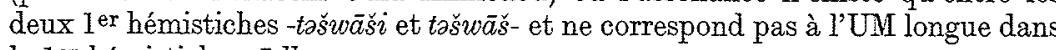
le $1^{\mathrm{er}}$ hémistiche $-s \bar{\imath} l l$ -

$z \partial z / t \smile \partial v$ tǎs $/ w \bar{a} / \tilde{s} \bar{\imath} \smile l l / \dot{g} \bar{a} / y e$

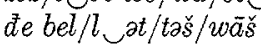

$r \bar{a} /{ }^{\circ} i \bar{d} \bar{\imath} / k \cup a t / t e y \check{s} / t \bar{a} / y e$

Ma tristesse ne connait plus de limites

Voilà certes des lieux évocateurs

Vois là-bas la Teychtaye

(u) $\ddot{d i}$ nab/kat lek/băš 13

Et ici la dune de Lekbach (des béliers).

Ces contraintes (rimes B en ṽev pour egilāl, en ṽc pour sgayyar) nous semblent constitutives des mètres la-bbeur et sjayyar. La raison en est, je pense, que le déséquilibre créé soit par la différence du nombre de pieds, soit par l'absence d'UM longue dans l'hémistiche de rime $B$, doit être compensé, faute de quoi le vers risquerait de perdre sa cohérence. Cette compensation paraît être fournie par la présence d'une voyelle longue là où l'autre hémistiche possède une UM longue (qui peut être, rappelons-le, constituée d'une syllabe du type cĩc ou du

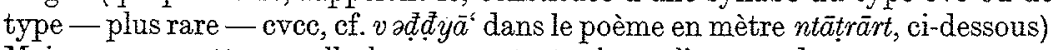
Mais comme cette voyelle longue porte toujours l'accent de mot, on peut se demander si l'accent n'est pas tout aussi important en soi que la voyelle longue. A mon avis le choix obligatoire d'une rime $B$ en $\bar{v} c v$ pour egilāl, en $\bar{v} c$ pour sgayyar sert à faire coïncider à chaque fois l'accent de mot à la rime avec l'accent métrique sur l'UM longue.

13 ' Et ' se prononce we devant deux consonnes et $\bar{u}$ devant cv. Lorsque $\bar{u}$ est un début d'un hémistiche, il ne compte pas comme UM. Une autre particularité à signaler, le déplacement possible des royelles. Ceci est très frequent, y compris dans la langage courant, pour la préposition ' $\mathrm{de}$ ' qui se transcrira tantôt man, tantôt amn, mais en poésie cette déplaçabilité de la voyelle es

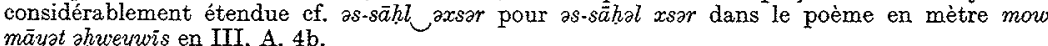

Je pense que l'absence de liberté dans le choix de la rime s'étend à des cas où les deux hémistiches ne diffèrent que par le nombre d'UM qu'ils comportent.
$7 \mathrm{UM}$
$6 \mathrm{UM}$
$7 \mathrm{UM}$ $h \bar{u} / n e m / r \bar{a} / d \cup i / s \bar{a} w / d \bar{u} / n \dot{ }$
Ici des maux m'assaillent man/hum 'ag/li vad̄ $\bar{d} / y \bar{a}^{5}$ wel/li man/hum nāw/šs/ni
Et j'en perds la raison,
Ils cherchent à me retenir ye/k $\bar{a} / n i$ nan/täăs $g \bar{a}$

Comme si je pouvais encore fuir.

Ce poème (donné par Md Maḥmūd wuld 'Abd al-Fattāh wuld Abyayr dan un manuscrit publié par Norris dans Folk lit., p. 163) est un exemple - le seul malheureusement dont je dispose - d'un mètre assez rare qui est une variante du mètre tīgadrīn: il s'en distingue seulement par l'absence d'une 7 ème UM dans le second hémistiche. Pour moi, ce n'est pas un hasard si le ler et le $3^{\text {ème }}$ hémistiches, qui comptent une UMI de plus que les 2 ème et 4 ème, ont une rime en $\bar{y} \mathrm{cv}$ et les autres, une rime en vic. Pour qu'il y ait coïncidence entre les deux accents de mot à la rime, il fallait en effet que l'accent tombe sur l'avant-

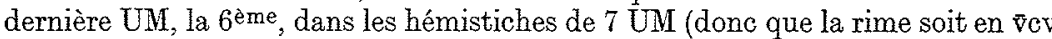
cf. -üni) et qu'il tombe par contre sur la dernière UM, la $6^{\text {ème }}$, dans les $2^{\text {ème }}$ et

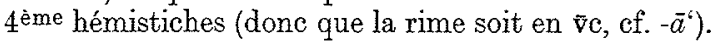

Si le rythme du vers est d'abord donné par l'opposition de quantité entre UM brèves et UM longues, on ne peut pas, à mon avis, sous-estimer le rôle complémentaire tenu par la rime et plus précisément, à travers la rime, l'accent de rime. Je pense que dans les mètres complexes l'accent de rime du $2^{\text {ème }}$ hémistiche doit coïncider, soit avec l'accent de rime du ler bémistiche, soit avec l'accent métrique tombant sur l'UM longue du ler hémistiche.

\section{Métrique et musique}

1. Tout le monde en Mauritanie sait que les rapports entre la poésie en hassäniyya ou gne et la musique maure sont étroits et le double sens de gine souligné par Aline Tauzin ("Autour de la poésie amoureuse maure de la Mauritanie et du Mali', Litt. Orale Arabo-Berbère, XII, 1982, p. 129) - à la fois 'poésie 'et 'musique' - et, dans une moindre mesure, celui de mganni - 'bon poète' mais aussi 'chantant' - ces polysémies donc ne sont pas fortuites, même si le poète n'est pas nécessairement un chanteur (tous les poètes ne sont pas des griots mais seuls les griots mettent en musique la poésie et la chantent).

La musique maure étant très complexe, le lecteur se réfèrera utilement aux spécialistes tels que Michel Guignard (Musique, honneur et plaisir au Sahara), je me limiterai ici au minimum nécessaire pour faire comprendre les rapports précis que les connaisseurs établissent entre telle forme poétique et tel 'type' de musique. La première distinction, la seule ou presque qui nous importe, est la distinction en cinq ' modes' (bhar, pl. bhür, qui - est-ce vraiment un hasard? - est le terme qui désigne les mètres de la poésie en arabe classique) - quatre modes seulement selon certains qui groupent sous la terme de saññ $\tilde{\imath}$ e les $3^{\text {ème }}$ et 4 ème modes.

Chaque mode est associé dans l'esprit des Maures à un certain nombre de sentiments :

A karr correspond le plaisir, la joie, le sentiment religieux. En l'écoutant, on pense à la jeunesse.

Fā giu excite, 'éveille le sang', la fierté, Ia colère, le désir de défendre une cause; il prépare à la guerre et à mourir. Il fait penser au premier âge viril.

Avec $l a-k h \bar{a} l, l a-b y \bar{a} \bar{d}$, nous entrons dans une zone de sentiments plus complexes et nuancés qui correspondent à l'âge mûr. Ils vont de la fierté dans $l a-k h \bar{a} l$ à l'amour et à la tristesse dans $l_{2}-b y \bar{a} \bar{d}$. 
Enfin la-btayt est un mode nostalgique et triste mais plus paisible: on pense au temps passé, à ses joies, mais aussi que tout cela doit avoir une fin (Guignard, op. cité, p. 92-3).

Chaque mode se divise ensuite en sous-modes qui ' colorent' le mode d'une façon particulière, du plus noir au plus blanc. 'La noirceur éveille l'enthousiasme, al-hamās. La blancheur enlève aux modes les plus violents une grande partie de leur force...' (idem, p. 96; voir également les pages 140-1-2).

Voyons maintenant les correspondances poésie et surtout métrique/musique telles qu'on les trouve sans variation majeure chez Leriche et chez Norris, correspondances que mes informateurs m'ont confirmées avec plus ou moins de précision.

(a) Au mode karr correspondent d'une part mreymìde, d'autre part $b \bar{u}$ 'amrān. mreymīde (2ème UM longue) se chante dans un sous-mode noir de karr? (appelé seyni karr) et bu' 'amrān (lère UM longue), dans un sous-mode blanc (mekke müse ou $l$-vāyzz) de karr.

(b) $v \bar{a} \dot{g} u$ est le mode dans lequel sont chantées les différentes variétés de l-bett $l_{2}-k b \vec{r} r$ ainsi que le theydin et le rasm (ici la correspondance se fait autant au niveau du genre - la poésie épique - qu'au niveau du mètre)

(c) la-khāl est le mode dans lequel se chante le mètre sgayyar à 5 ème UM longue (et, selon un informateur, le mètre tìgadrìn).

(d) la-byāt est celui dans lequel se chante le mètre la-bḅeyr ou ḅbeyr egila $a l$ à 6 ème UM longue.

(e) Quant au cinquième mode, il est tellement associé au mètre la-bteyt (aucune UM longue dans l'hémistiche) qu'on lui donne fréquemment ce nom, en particulier en Adrār et à l'Est, alors qu'au Trārze il s'appelle beygi 'le petit restant'.

2. Ces correspondances sont connues, mais les raisons techniques de ces relations n'ont jamais, à ma connaissance, été exposées. C'est ce que je voudrais tenter de faire maintenant, en m'excusant de ne pouvoir intégrer ici l'ensemble de la poésie chantée sur le mode vāgu (qui est à la fois trop riche et trop mal connu jusqu'à présent).

Les métriciens arabes ont distingué quatre éléments métriques, d'une part les $a s b \bar{a} b$ litt. 'cordes' (le sabab 'léger' cve ou- et le $s a b a b$ 'lourd ' cvev ou uu), d'autre part les awtäd litt. 'piquets, piliers' (le watid mažmū' litt. " piquet uni', cveve ou u- et le watid mafrúq litt. "piquet dispersé', cvecv ou -u). Gotthold Weil, l'auteur de l'article 'arúd dans l'Encyclopédie de l'Islam (I, p. 688-8) estime que les syllabes longues des awtād portent un accent métrique et que le mètre a dono un rythme ascendant lorsque son noyau rythmique est le watid mažm $\bar{u}^{-} u<$ et un rythme descendant lorsque son noyau rythmique est le watid

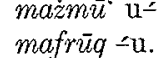

Sa démonstration ne convainct pas tous les spécialistes, beaucoup refusant d'accorder un rôle à l'accent dans la métrique arabe (leur premier argument étant que ni les grammairiens ni les métriciens arabes n'en ont tenu compte). D'autres, tel Georges Bohas, tout en critiquant l'argumentation de Weil, le rejoignent sur sa conclusion et jugent fondée sa distinction entre les séquences simples UM brève + UM longue ou l'inverse, $u-$ ou -u, et les suites marquées uet -u ('Watid. Définitions, discussions et perspectives', Analyses-Théorie, $1980-81,55-81)$

Partant de cette conclusion et du fait qu'il existe dans le gne une opposition comparable entre des UM brèves et des UM longues d'une part, un accent métrique sur les ONI longues d'autre part, je ferai l'hypothèse qu'il existe dans le gne comme dans la poésie en arabe classique, non pas seulement des UM longues isolées mais des autād c'est à dire des éléments associant à l'UM longue accentuée, une UM brève.

Selon cette hypothèse, mreymīde s'oppose à bū 'amrān par la nature du watid: watid mažmü, done rythme ascendant, dans mreymïde (u-uuuuu/-) watid mafrü $q$, donc rythme descendant, dans bü' amrān (-uuuuuu/-).

De même, on trouve un watid mazm $\bar{u}^{-}$, donc un rythme ascendant, dans sgayyar (uuuu-uu/-, uuuu-) ${ }^{14}$ et un watid mafrüq, donc un rythme descendant, dans la-ḅbeyr (unuuu-u, unuuuuu).

Les relations entre les mètres du gne et les modes et sous-modes musicaux deviennent alors compréhensibles. mreymìde correspondant au sous-mode noir de karr et sjayyar au sous-mode noir de sañ ñime (ou mode la-khäl 'La noirceur') d'une part, bü 'amrān correspondant au sous-mode blane de kary et la-bbeyr au sous-mode blanc de saññme (ou mode la-byāố "la blancheur') d'autre part, il s'établit une relation double entre:

- le rythme ascendant et la noirceur,

- le rythme descendant et la blancheur,

ce qui corrobore parfaitement la définition donnée par Guignard de la noirceur et de la blancheur en musique maure.

Par ailleurs, les relations de mrezmīde et de $b \bar{u}$ "amrān avec le mode karr (expression de la joie, du plaisir) d'un côté, celles de sgayyzar et de la-bbeyr avec les modes la-khāl et la-byä (expression de sentiments plus nuancés) d'un autre côté, peuvent aisément s'expliquer par le déplacement du watid dans l'hémistiche, comme dans les mètres de la poésie classique: ' $(\alpha)$ le pied commence immédiatement au noyau rythmique, ce qui crée un rythme ascendant particulièrement fort: $u \leq x, u \leq x x, u-u u_{-}^{-} ;(b)$ le noyau est à la fin du pied, ce qui donne au rythme une allure une peu pressée et sautillante: $x^{\prime}-x^{\prime}, x^{-}-$, uu-u- ; $(c)$ le noyau est à l'intérieur du pied, ce qui entrave un peu la force du rythme ascendant: xu<x ('Article 'arüd', EL, I, p. 696)

Pour Weil, on a donc des mètres classiques qui développent plus que les autres le noyau du rythme ascendant $u=$, tels que tawoìl, basìt, wäfir et kămil, et d'autres mètres tels que sarī et xafï qui comportent au contraire un watid mafrüq $\sim \mathrm{u}$, ccur du rythme descendant.

3. On serait en droit de se demander comment les principes établis pour les mètres classiques peuvent être valables pour les mètres maures, en l'absence de toute relation entre les mètres classiques et les mètres maures. Cependant cette relation existe bien dans quelques cas, d'après les deux manuscrits publiés en annexe dans Folk lit. Selon leurs auteurs, en effet, mreymide correspond au mètre classique wāfir, la-bbeyr à xafī $f$ et la-bteyt à tawīl. (Le second auteur, avec quelque circonspection, s'en remet à l'autorité d'un poète qui a mis en vers ces correspondances cf. Norris, Folk lit., p. 190-91.) Ces relations sont comprises actuellement, d'après les informateurs interrogés, comme une coïncidence passant par la musique: si le correspondant de la-bteyt est tawill, c'est parce que les deux mètres se chantent dans le mode beygi ou la-bteyt. Dans le cas précis de la-bteyt, je rejoindrai, au moins provisoirement, cette interprétation qui est

14 Dans la mesure où le second hémistiche s'arrête après la sème UM longue, il semble logique de poser que le watid comprend les 4 ème et 5 ème CMI longues (et non les 5 et beme pieds, oo

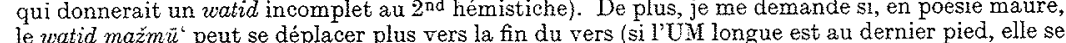
confond avec la rime). On notera que dans le mètre wăkdi, l'un des la-btüt la-kbär à avoir deux VM longues fixes, on a denx wotid l'un au début comme dans mreymide et l'autre avant les deux dernières UM, comme dans sgagyyər: u二uuuuu=uu, ce qui renforce notre hypothèse d'un watid mażm $\tilde{u}^{\prime}$ dans sğayyar. 
d'ailleurs aussi celle de Norris (Folk lit., note 8 de la page 159). Les deux autres cas me semblent tout à fait différents puisqu'on peut déjà affirmer que mreymìdo a un rythme ascendant comme wäfr et la-bbeyr, un rythme descendant comme xaf $2 f$.

De plus, d'après notre hypothèse et en notant les mètres classiques conformément à Weil ( $E I, \mathrm{x}, \mathrm{p} .690$ ), mreymïde et wäfir commencent tous les deux par un watid mažmǘ, la-ḅbeyr et xaf̄̃ f ont chacun un watid mafrüq après la 4 ème UM:

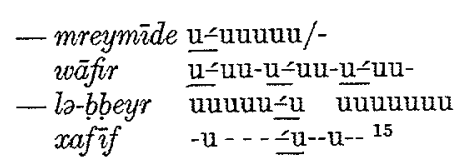

Indépendamment ou plutôt en complémentarité avee une dérivation des formes du ğne à partir de la poésie classique passant par al-bett la-kbīr, telle que l'envisage Ahmed-Bâba Miské (Al-Wasît, p. 53-4), je pense qu'on peut expliquer nombre de caractéristiques de la métrique maure par une autre voie, en prenant en considération les éléments communs et les éléments divergents de chacun de ces deux couples de mètres.

Si la caractéristique commune entre mreymäde et wäfir d'une part, la-ḅbeyr et xaf if d'autre part, est le watid (le $1^{\text {er }}$ watid dans le cas du mètre classique quand il y en a plusieurs), sa nature (mažmü ou mafrüq) et sa place, plusieurs conséquences en découlent. D'abord cela rend très féconde l'hypothèse selon laquelle l'accent métrique est important en arabe classique et distingue bien le watid d'une suite quelconque de deux syllabes, l'une brève, l'autre longue. Cela signifie ensuite que le gne a perdu la distinction entre UM brève et UM longue à l'intérieur des hémistiches, sauf dans les awtād, ce qui revient à dire qu'elle s'est maintenue seulement là où il y avait un accent métrique. Il apparaît enfin que le nombre d'UM par vers est secondaire en dehors du fait qu'il doit être fixe (et que des hémistiches de longueur inégale doivent compenser leur irrégularité par une contrainte accrue sur la rime ef. chap. III)

'Secondaire' ne veut pas dire négligeable, bien évidemment, mais implique que des mètres de longueur inégale, ayant un watid ou une absence de watid en commun (cas des mètres la-bteyt, par exemple) sont beaucoup plus proches les uns des autres que des mètres qui ne partagent que le nombre d'UM, comme

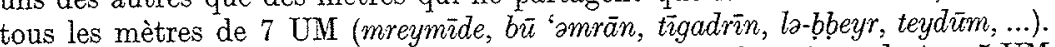
On peut ainsi supposer, avec peu de risque d'erreur, que les mètres de 4 ou 5 UM à 2 ème UM lonoue (le 1er s'appelle mowmāyat ahweywiss, cf. I, A, 4 et 5) sont des variantes de mreymìde et se chantent également dans le sous-mode noir de karr.

Dans la poésie en arabe classique, comme dans le ġne, les variations de longueur des mètres sont possibles et constituent même "la déviation la plus minime' ( $E I$, I, p. 691). La seule différence est qu'un mètre comme wäfir ne se raccourcit pas librement mais par la suppression de séquences longues appelées $a \check{z} \bar{a}^{c}$ (ne comportant jamais plus d'un watid), ce qui donne:

$w \bar{a} f i r$

ou même

u-uu-u<umuu<uu-

úuu-úuu- (réduction d'un tiers)

úu $\quad$ (réduction de deux tiers)

${ }^{15}$ On ne s'étonnera pas, d'après notre interprétation, de ne pas avoir de correspondant classique à bü 'amrān-uuuuuu// car aucun mètre classique ne peut commencer par un watid mafrüq, p. 76$)$.
Notons que la première réduction donne quasiment la mètre maure que Leriche appelle 'ațše et dont il présente l'hémistiche (du type u-uuuu-uuu) comme étant composé de deux hémistiches du mètre de 5 UM à 2 ème UM longue u-uuu ('Poésie et musique maure', p. 720-21); mais on peut se demander si, historiquement, 'aťše n'a pas été la première étape de transition à partir de $w \bar{a} f i r$.

4. J'ai cherché à montrer, à travers une analyse de la métrique, comment pouvait se justifier l'association de tel mètre du ğne avec tel sous-mode de tel mode musical, chaque mode et chaque sous-mode correspondant selon Guignard à l'expression d'une certaine gamme de sentiments. Il serait intéressant de savoir si le contenu des poèmes varie selon le mètre adopté et si ce contenu est identique à celui de la forme musicale correspondante. Sur ce point, l'inventaire de tous les mètres utilisés dans notre corpus de référence conduit immédiatement à des constatations significatives.

- Les mètres mreymīde et bü 'amrān ne sont jamais utilisés dans la poésie amoureuse (du moins dans les 115 poèmes amoureux donnés par El Kacem Ould Ahmedou, Md Ould Ahmedou Bamba, Dustin Cowell et Aline Tauzin). - Toujours d'après ce corpus de 115 poèmes amoureux, $5 \%$ des poèmes sont dans le mètre sgayyar, $7 \%$ dans le mètre b̆beyr egitāl et $88 \%$ dans les mètres la-bteyt. Le pourcentage varie naturellement selon les poètes. Ainsi, la totalité des poèmes de M'hammed Ould Ahmed Youra constituant le corpus de Md Ould Ahmedou Bamba est composée dans la-bteyt: la-bteyt correspond bien en effet au caractère nostalgique de la poésie amoureuse de ce poète.

- Dans la poésie de la résistance présentée par Md Ould Boyah, les mètres les plus fréquents sont encore les mètres la-bteyt au sens large. Le seul poème en mètre sğayyar est un petit gãv alerte critiquant l'inféodation de l'assemblée aux colonialistes (poème 21). Deux des trois poèmes en mètre bbeyr egilāl sont d'un ton plus nostalgique et commencent par une invocation (ya kadyat-ne "ô notre montagne...', ya mulāne 'ô notre Maître...'). Le mètre bü 'amrān n'est toujours pas représenté mais quatre poèmes (sur 48) sont dans le mètre mreymīde. L'un d'eux, composé à la gloire de l'émir Bekkār Ould Sweyd Ahmed, mort en résistänt à la colonisation, est certainement assez représentatif de la poésie en mètre mreymìde, tout comme cet échange de poèmes dans le même mètre qui figure dans l'ouvrage de David Cohen (Le parler arabe hassänãyo de Mauritanie, p. 236-9) et dont le ton est donné dès le premier hémistiche:

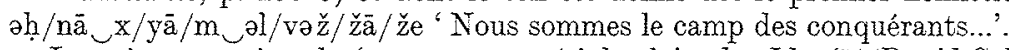

- Le poème en mètre bū 'amrān composé à la gloire des Idaw' $\imath \bar{s}$ (David Cohen, idem, p. 242-3) exprime lui-aussi des sentiments de fierté, cependant c'est une fierté moins combattive, qui ne naît pas d'une confrontation avec des ennemis mais de la reconnaissance de qualités intrinsèques, de courage et de puissance.

Les résultats vont donc dans le sens de ce qu'on pouvait attendre, sans pour autant établir des barrières absolues entre les usages des différents mètres.

Si la-bteyt at-tāmm (de $8 \mathrm{UM}$ ) et, secondairement, la-bteyt on-nāqaș (de $6 \mathrm{UM}$ ) constituent dans tous les cas les mètres les plus utilisés, c'est à mon avis parce que leur rythme, moins marqué dans un sens comme dans l'autre (absence de watid), convient plus ou moins à l'expression de sentiments très divers: la-bteyt serait à la fois le mètre exprimant la nostalgie et le 'degré zéro' de la métrique, celui par rapport auquel tous les autres mètres marqués. On peut alors se demander si la correspondance la-bteyt/taw ne se justifie pas simplement comme la mise en relation des deux mètres qui servent de référence à tous les autres - remarquons que tawīl est le premier mètre du premier cercle dans la théorie d'Al-Xalill (cf. EI, I, p. 690). 


\section{Conclusion}

La métrique et la rime concourent à modeler les vers du gine, la poésie en dialecte hassāniyya.

Les différents mètres se caractérisent par le nombre et la nature des unités métriques (UM), ce qui donne un rythme de versification basée, comme dans la poésie en arabe classique, sur une opposition de quantité. L'opposition entre UM brève et UMI longue s'est cependant déplacée par rapport au classique : de l'opposition $\mathrm{cv} / \mathrm{c} \overline{\mathrm{v}}$ ou cve on est passé à l'opposition $\mathrm{ev}, \mathrm{c} \overline{\mathrm{v}}$ ou $\mathrm{evc} / \mathrm{c} \overline{\mathrm{v}} \mathrm{c}$ ou $\mathrm{crce}$ du fait des différences entre la structure syllabique du dialecte et celle du classique. A un premier groupe de mètres sans UM longue dans l'hémistiche, que certains désignent collectivement par la-bteyt ("le petit mètre'), s'oppose une série d'autres mètres qui se différencient les uns des autres par la place de leur UM longue (les mètres comptant deux UMI longues sont peu nombreux et en général très peu usités). Sur cette UM longue tombe un accent métrique d'intonation qui a pour effet de redonner aux voyelles de fin de mot leur quantité de longues.

Les rimes sont variées, les plus fréquentes sont du type $\bar{v} c$ et $\bar{v} c r$, mais elles peuvent se réduire à une consonne seule. L'alternance des rimes dépend de la forme poétique choisie (A B, A B, pour le gà $v$, A A A B A B(...) pour la tal'a etc.). Le choix des rimes est en général indépendant du mètre, sauf lorsque les bémistiches ne sont pas composés dans le même mètre. En effet, dans le cas des mètres complexes, les types des rimes (en général $B$ seul, parfois $A$ et $B$ ) sont fixes, par exemple v̄c pour le mètre sjayyar et v̄ev pour le mètre la-bbeyr, cec afin que l'accent sur la rime B tombe sur le même pied que l'accent sur la rime A ou coïncide avec l'accent métrique porté par l'UM longue de l'hémistiche de rime $\mathrm{A}$.

Si l'on pose que le rythme du vers repose, comme en arabe classique, sur la présence d'unités supérieures à la syllabe, les awtād ( $\mathrm{u}<$ et $\sim \mathrm{u}$ ), on peut expliquer toutes les relations que les connaisseurs établissent entre la poésie et la musique maures d'une part, les mètres du gine et les mètres du classique d'autre part. On constate en particulier que:

- le sous-mode noir correspond au rythme ascendant (présence d'un watid $\left.m a z ̌ m \bar{u} u^{-} u^{\prime}\right)$

- le sous-mode blanc correspond au rythme descendant (présence d'un watid mafrūq $\sim \mathrm{u}$ ),

- le mode karr correspond aux mètres ayant un watid au début de l'hémistiche, - le mode saññme correspond aux mètres ayant un watid à la fin de l'hémistiche,

- le mode la-bteyt ou beygi correspond aux mètres sans watid dans l'hémistiche.

A chaque sous-mode de chaque mode on fait traditionnellement correspondre une certaine gamme de sentiments, on pouvait done s'attendre à ce que le choix du mètre ne soit pas sans rapport avec le contenu du poème. Les sondages que j'ai effectués semblent bien indiquer une variation du contenu poétique en rapport avec le mètre choisi, mais on notera que cette spécialisation des mètres est limitée par la tendance à employer les mètres non marqués du genre la-bteyt.

Pour Ahmed-Bâba Miské il ne faisait pas de doute que le gne descendait en droite ligne du $s^{\prime} i^{\prime} r$, la poésie en arabe classique. Mon analyse me conduit à la même conclusion mais en fonction de considérations différentes, basées essentiellement sur la métrique. Dans les mètres du gne on retrouve en effet les caractéristiques principales de la métrique classique (rythme de quantité variant en fonction de la nature et de la place des awtād, accent métrique sur le
UM longues des awtād, variation possible du nombre de pieds). Les différences sont certes importantes mais affectent des caractéristiques secondaires du système métrique classique (l'existence d'une opposition brève/longue en dehors des awt $\bar{a} \bar{d}$, le nombre des UM par mètre, sans parler du déplacement de l'opposition brève/longue que j'ai déjà décrit).

Le système métrique du gine dériverait donc du système métrique du classique mais on ne peut comprendre la dérivation du premier à partir du second qu'en prenant en considération le rôle de l'accent métrique.

Cette probable dérivation du gne à partir du šír $r$ ne préjuge pas cependant de l'existence d'influences diverses (comme celle de la poésie arabe andalouse) qui ont pu s'exercer parallèlement et sont peut-être plus sensibles dans d'autres domaines de la poésie maure (formes poétiques, thèmes, etc.).

\section{BIBLIOGRAPHIE}

Awad, Georges et Bohas, Georges. ' Note à propos des sur-lourdes en arabe clasșique ', Analyses, Théorie, $1980-81$, p. $60-6 \tilde{5}$

Ben Alhousseñni, Hamid. A perçu sur la poésie maure del'Azaonad (région de Tombouctou) - Mali, Bonéoté, $43 \mathrm{p}$. Bohas, Georges.

Cantineau, Jean. 'Cours de phonétique arabe, p. 1 à 125 , ' Esquisse d'une phonologie de l'arabe classique ', p. 165 à 204, Etudes de linguistique arabe, Paris, Klincksieck, 1960 .

Cohen, David. Le parler arabe kassāninya de Mauritanie, Paris, Klincksieck, 1963.

Encyclopédie de l'Islam (nouvelle édition), Leiden, Brill, Paris/G. P. Maisonneuve \& Larose 'Article 'arüd', $\mathrm{x}, 1975$, p. 688-98, de Gotthold Weil; "Article haraka wa-sukün de Moh. Ben Cheneb et S. A. Bonebakker.

Garde, Paul. L'accent, Coll. S.U.P., Paris, P.U.F., 1968.

Guignard, Michel. Musique, honneur et plaisir au Sahara, Paris, Lib. Geuthner, 1975. Leriche, Albert. 'Poésie et musique maure', Bull. IFAN, t.xir, Dakar, 1950, p. 710-43. Mehiri, Abdelkader. Les theories grammaticales d' Lon Jinn, Publ. de I' niv. de Tunis, 1973. sieck, 1970

Norris, H. T. (Folk Lit.) Shinquiti folk literature and song, Oxford, Clarendon Press, 1968 .

ss ta dir. de Y. Guider, 1981.
Ould Ahmedou Bamba, Mohammed. Mammed Ould Ahmed Youra, poète amoureux, Mémoire de

l'E.N.S., ss la dir. de Y. Guider, 1982 .
Onld ss la dir. de Y. Guider, 1982.

Ould Hasni, Moulaye Ahmed. La poésieépique hassane du Theydin, Mémoire de l'E.N.S., ss la dir. de Y. Guider, 1981 .

Ould Youra, M. Abderrahim. La joute poétique dans la poésie de Sid'Ahmed O. Ahmed O. Aìda Mémoire de l'E.N.S., ss la dir. de Y. Guider, 1982.

a amoureuse maure de la Mauritanie et du Mali ', Littéraiure Orale Arabo-Berbere, Bull. 13, Paris, 1982, p. 129-46. 